Check for updates

Cite this: Chem. Commun., 2021,

57, 1631

Received 22nd December 2020,

Accepted 11th January 2021

DOI: $10.1039 / \mathrm{d} 0 \mathrm{cc} 08310 \mathrm{k}$

rsc.li/chemcomm

\section{Cascade communication in disordered networks of enzyme-loaded microdroplets $\dagger$}

\author{
Marko Pavlovic, Markus Antonietti (D) and Lukas Zeininger (D) *
}

\begin{abstract}
A network of aqueous emulsion droplets that exhibits programmed and directional chemical inter-droplet communication is described. A non-reciprocal transfer of substrates between enzyme-containing aqueous emulsion droplets is realized by (biochemically) induced osmolarity gradients and concomitant concentration gradients are used to direct a multistep enzymatic cascade reaction across multiple droplets.
\end{abstract}

Regulation of chemical reactivity in reaction cascades is critical to the functioning and survival of living organisms, ${ }^{1,2}$ and striking examples include the metabolization of sugars for cellular energy supply, or the immune system's capability to specifically respond to perpetrators by transforming chemical recognition events into the release of inflammatory counteragents. In nature, an asymmetry in chemical communication is often achieved by compartmentalizing active species, e.g. enzymes, within cells or subcellular organelles. ${ }^{3,4}$

Spatial separation and positional assembly is to a large extend responsible for the regulation and associated modulation of chemical reactivity, for minimizing the diffusion pathways of intermediates between enzymes, and for the overall efficiency and specificity of biological reactions. ${ }^{5-9}$ Also, rivalling reaction centers have to be spatially separated from one another in order to control their destructive interference. ${ }^{10-12}$ Whereas a direct mimicking of the complexity of living cells cannot yet be realized in artificial assemblies, a dissection and emulation of these individual capabilities within a simplified synthetic system that shows similarly sophisticated behavior would be of large scientific and technological value.

A key to the realization of artificial autonomously communicating colloidal objects is a trigger-induced evoking of a nonequilibrium state, for example a chemical concentration gradient

Department of Colloid Chemistry, Max Planck Institute of Colloids and Interfaces, Am Muehlenberg 1, 14476 Potsdam, Germany.

E-mail: lukas.zeininger@mpikg.mpg.de

$\dagger$ Electronic supplementary information (ESI) available: Materials and methods, experimental details. See DOI: 10.1039/d0cc08310k that can act as a driving force to induce a non-reciprocal masstransfer and start a chemical reaction cascade. ${ }^{13,14}$ For this purpose, the generation of stable but active colloidal subdivided systems in thermodynamically permissible out-of-equilibrium states is required. In this context, emulsion droplets, kinetically stabilized dispersions of fluids in another phase-separated fluid medium, offer a versatile platform because they are thermodynamically metastable, highly dynamic, while their stability can be influenced by a variety of chemical and physical triggers. ${ }^{15-18}$ The dynamic liquid interfaces in surfactant stabilized emulsion droplets, in which molecules are continually exchanged between droplets and the continuous phase, closely resembles the environment found at cell surfaces. ${ }^{19-21}$ In aqueous emulsion droplets, a dynamic solvent exchange and mass transfer between droplets is often associated with osmotic pressure imbalances, ${ }^{22-25}$ which offers rich opportunities to study nonequilibrium interactions between these liquid colloidal microcontainers.

In this paper, we report on a directional transfer of chemical information across disordered networks of enzyme-containing aqueous micro-droplets. An asymmetry in the communication between the aqueous microcontainers was actuated by (biochemically) induced osmotic pressure imbalances (Fig. 1). Arising chemical concentration gradients were used to evoke and accelerate non-reciprocal transfer of substrates between individual droplets. Through compartmentalization of enzymes within the individual aqueous microcontainers, a biochemically mediated artificial communication cascade across multiple emulsion droplets was realized.

Emulsions represent highly dynamic systems and mass exchange continuously occurs between droplets and the continuous phase. We were interested in droplets of different composition to investigate a possible directional mass transfer. To this end, we prepared aqueous droplets containing different concentrations of an osmolyte (glucose) stabilized by the same surfactant and studied the solvent flux between the droplets as a function of the osmolyte concentration difference. Micrometer-sized emulsion droplets $\left(d_{\mathrm{avg}}=120 \mu \mathrm{m}\right)$ were 


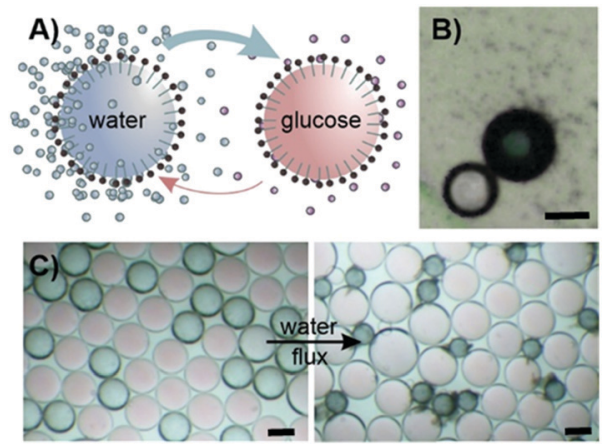

Fig. 1 Osmotic pressure-driven non-reciprocal solvent transfer between aqueous microdroplets of different osmolyte concentrations (blue droplets: 0 wt\% and red droplets: 20 wt\% D-glucose) (A) schematic sketch displaying the non-uniform distribution of nanoemulsions (small droplets) formed via secondary emulsification. Arrows indicate the associated net asymmetric solvent exchange between the two types of droplets. (B) Micrograph displaying the asymmetric distribution of nanoemulsions surrounding such droplet pairs. (C) Overall solvent flux start and end ( $t=3 \mathrm{~h}$ ) point of an osmotic pressure imbalance-driven solvent transfer.

formed by emulsifying an aqueous sample solution within a hydrocarbon oil (tetradecane) containing Span 80 inside a microfluidic chip. Directly after generation of the droplets, spontaneous secondary emulsification occurred, i.e. small nano-sized droplets are formed near the interface. ${ }^{26-28}$ While all big droplets shrank in size due to this spontaneous emulsification process, the formation of nano-emulsion droplets was different in rate, with lower osmotic pressure droplets exhibiting significantly higher productivity.

When placing two droplets of different osmotic pressure next to each other, an overall non-reciprocal solvent exchange occurred between the two droplets via the continuous phase (Fig. 1), potentially driven by the asymmetric formation and distribution of nanoemulsions around the droplets. Due to the osmolarity gradient between the droplets, we observed an overall solvent flux from the hypotonic to the hypertonic region until an equilibrium in osmotic pressure was reached. As displayed in Fig. 1C, the solvent exchange could be readily monitored by following the concomitant size variations of the droplets.
The extend and directionality of this solvent exchange between aqueous emulsion droplets was determined and could be modulated by multiple factors, including the surfactant type, surfactant concentration, and the droplets' osmotic pressure. We started our investigations with a combination of droplets containing 0 and $30 \mathrm{wt} \%$ glucose, thus exhibiting a large osmotic pressure difference, and studied the solvent exchange rates. Depending on the hydrophilicity of the employed Span surfactants, an increased secondary emulsification rate and thus a faster mass transfer between the droplets was observed. While droplets stabilized by Span 20, a surfactant with a higher hydrophilic-lipophilic balance (HLB) value of 8.9, reached equilibrium after about $t \sim 1.5 \mathrm{~h}$, this exchange rate was significantly prolonged in Span 80 (HLB: 4.3) and Span 85 (HLB: 1.8) solutions to $t=3 \mathrm{~h}$ and $t=4 \mathrm{~h}$, respectively (Fig. 2A). The water flux between the droplets could further be influenced as a function of the surfactant concentration in the continuous phase. A decrease of the Span concentration from $4 \mathrm{wt} \%$ down to $1 \mathrm{wt} \%$ led to a lowered micellar solubilization of the dispersed phase and thus resulted in decreased equilibration rates (Fig. 2B). In all experiments, the solvent flux between the droplets was accompanied by molecular diffusion of water through the continuous oil phase, which however is considered to be low.

Next, we investigated the influence of the osmolarity gradient between the droplets on the asymmetric solvent exchange (Fig. 2C). Here, lowering the osmolyte concentration within the hypertonic droplets resulted in a decrease in the overall volume variation of the droplets, as a directional mass transfer between the droplets only occurred until an equilibrium in osmotic pressure was reached. Interestingly, through the presence of an osmolyte within all droplets, both the extent and the rate of directional solvent flux could be significantly influenced (Fig. 2D). In addition to the extent of the directional mass exchange, the presence of osmolytes at differing concentrations within both droplets led to a decrease in the rate of the spontaneous secondary emulsification thereby lowering the speed of the solvent exchange.

Within these droplet combinations, the directional solvent flux started immediately after placing the droplets next to each
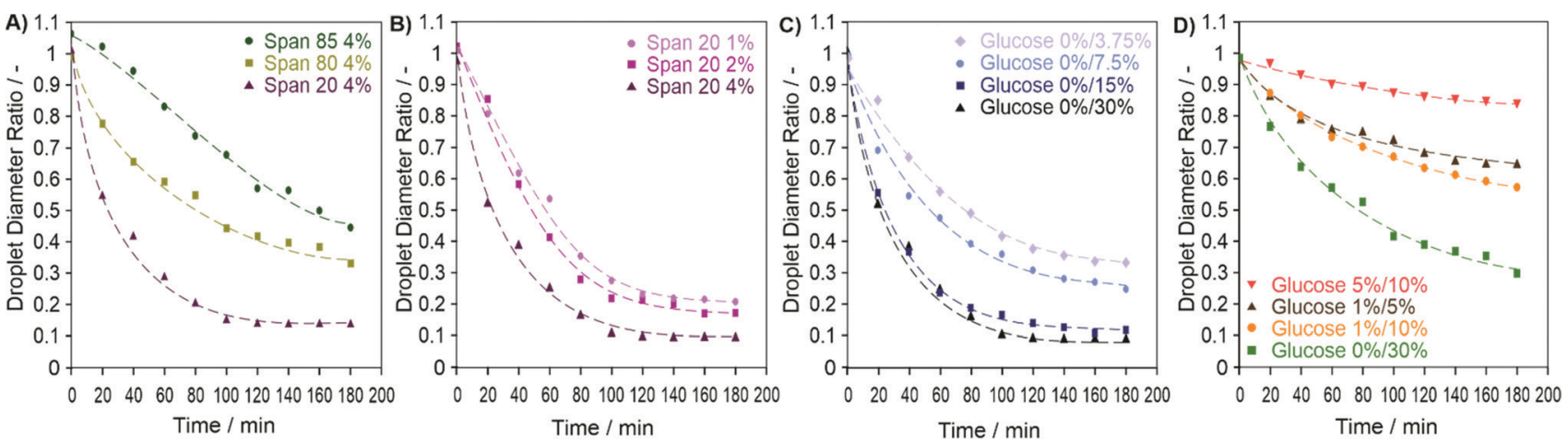

Fig. 2 Dependency of the osmotic pressure driven directional solvent flux between aqueous microdroplets on (A) the surfactant type (droplets contained $0 \%$ and $30 \%$ wt $\%$ D-glucose); (B) the surfactant concentration (droplets: $0 \%$ and $30 \%$ wt $\%$ D-glucose); (C) the osmotic pressure difference (performed in 4 wt\% Span 80 solution); and (D) the osmolyte concentrations within the individual droplets (4 wt\% Span 80 ). 
other and proceeded until an equilibrium in osmotic pressure was reached. In order to evoke dynamic changes in the directional mass transfer between two droplets, we next investigated whether a biochemical reaction could induce such an out-ofequilibrium state and thus, a non-reciprocal inter-droplet communication. Therefore, we first prepared two sets of aqueous emulsions, both of which contained the disaccharide sucrose as osmolyte in the same concentrations. Droplets of this type display a non-directional, continuous exchange of solvent. However, when additionally encapsulating the enzyme invertase into one set of droplets, a biochemically induced directional asymmetric solvent flux between the droplets was induced (Fig. 3A). Hydrolysis of sucrose into fructose and glucose within the confined space of one of the two droplets caused an increase in osmotic pressure thereby evoking an osmolarity gradient between the droplets. ${ }^{29,30}$ The enzymatically triggered directional mass transfer from one droplet to another was monitored by tracking the size variations of droplets containing different starting concentrations of sucrose over time.

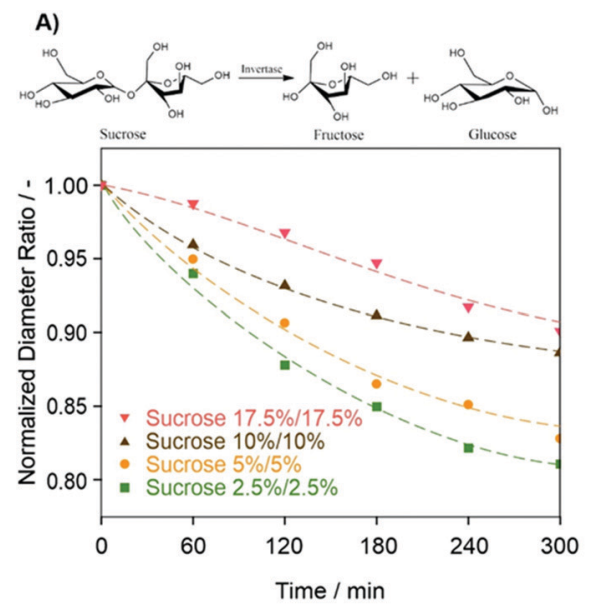

B)
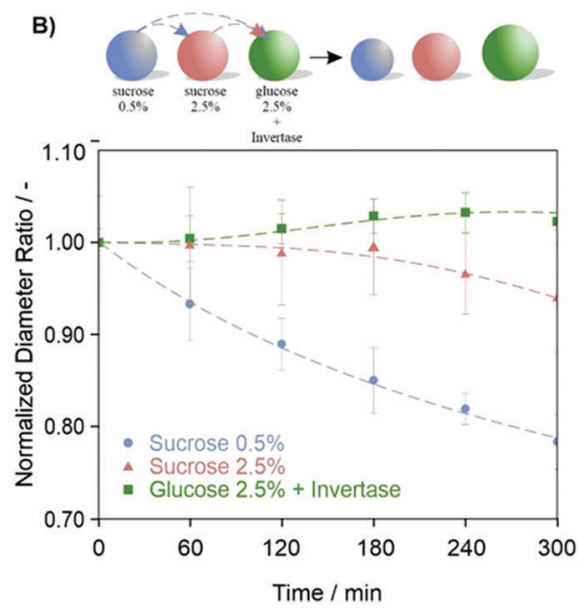

Fig. 3 Bio-chemically induced directional mass transport between aqueous emulsion droplets. (A) Mass transfer between two droplets induced by invertase triggered osmotic pressure gradients; (B) mass transfer cascade within arrays of three droplets of different composition by combining static and induced osmotic pressure differences.
We anticipated that a biochemically induced directional mass transfer could serve as a control unit to direct the transfer of chemical substrates between multiple droplets. To test this hypothesis, three types of droplets of different composition were placed together in order to establish a solvent transfer cascade. We first placed two types of droplets with a matched osmotic pressure, one containing sucrose (2.5 wt\%) and the other containing glucose $(2.5 \mathrm{wt} \%)$ and invertase into a sample holder. Then, a transfer cascade was actuated by adding droplets containing lower concentrations of sucrose $(0.5 \mathrm{wt} \%)$ (Fig. 3B). It is known that upon spontaneous interface emulsification, small molecular components can partition within nanodroplets, whereas very hydrophilic compounds, higher molecular weight polymers, and enzymes remain inside the macroemulsion droplets. ${ }^{31,32}$ Thus, in our scenario, small contents of sucrose molecules could be transferred from the actuating 'sacrificial' droplet to the second and the third invertase-containing droplets thereby inducing a further increase in osmotic pressure of the latter. As a result, following an initial rapid solvent flux from the actuating droplets towards the other two sets of droplets, a subsequent solvent flux from the second to the third droplet was initiated by the biochemically triggered osmotic pressure increase in the latter, which could be followed by a swelling of the invertase-containing droplets in this system.

Having established an understanding of both static and biochemically induced directional mass transfer within networks of aqueous emulsion droplets of different composition we next set out to leverage these findings for realizing a multistep chemical cascade reaction across multiple emulsion droplets invoked and mediated by the parallel and independent performance of four different encapsulated enzymes. More specifically, we investigated a three-step biochemical transformation of a substrate (xanthine) into the release of a fluorescent marker (resorufin), in which a directional transfer of substrates and intermediates between the individual enzymatic reaction center-containing droplets was simultaneously guided by invertase-mediated osmotic pressure imbalances between the droplets (Fig. 4). The reaction cascade was actuated by the placement of a xanthine-containing aqueous sucrose droplets (0.5 wt\%). A directional solvent transfer to droplets of higher osmotic pressure was used to efficiently transfer the substrate xanthine. Compounds of lower hydrophilicity such as xanthine preferably partition within the nano-droplet transporters and were therefore efficiently transferred instead of increasing their concentration inside the starting droplet. ${ }^{31,32}$ Encapsulated xanthine oxidase within droplets of higher sucrose concentrations $(2.0 \mathrm{wt} \%)$ produced uric acid as an intermediate reaction product. In parallel, the simultaneous transfer of sucrose to a third type of droplets comprised of glucose (2.5 wt\%) and invertase invoked a steady increase in their osmotic pressure thereby directing the solvent flux towards them. A directional transfer of uric acid to the final droplets enabled an uricasemediated transformation into allantoin, which generated hydrogen peroxide as a byproduct. The latter was consumed by horseradish peroxidase which then catalyzed the transformation of amplex red into the fluorescent marker resorufin. 


\section{A)}

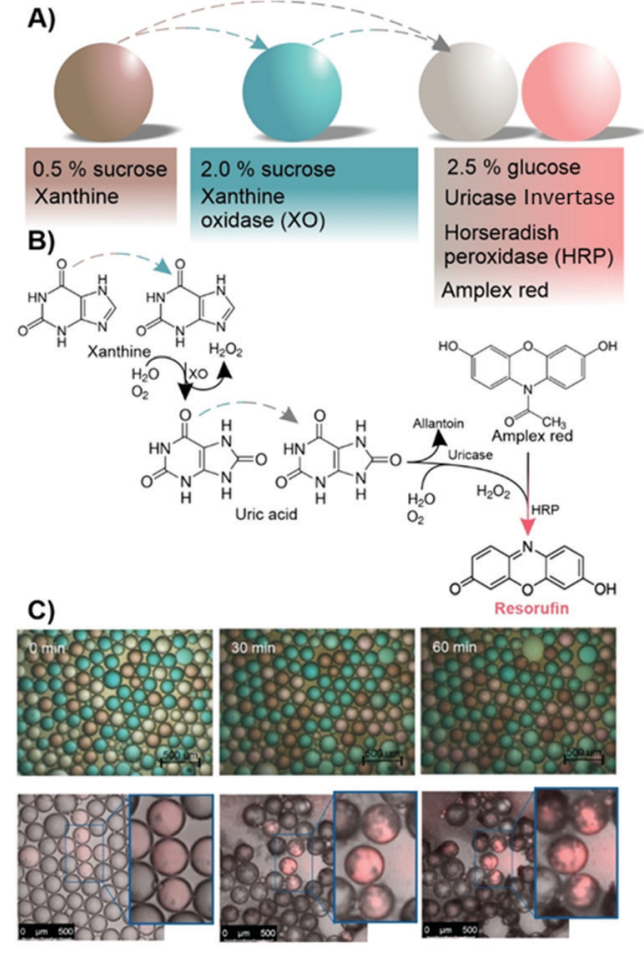

Fig. 4 Enzymatic reaction cascade across multiple droplets coupled to a regulatory biochemical reaction triggering an osmotic imbalance driven non-reciprocal mass transfer of substrates between the droplets: (A) schematic representation of the droplet cascade; $(B)$ reaction scheme outlining both the enzymatic transformation and the transfer of substrates between droplets; (C) time-dependent evolution of the cascade reaction progress tracked via optical and fluorescence micrographs of the droplet arrays.

Reference experiments showed that the progress of the chemical cascade reaction was reliant on the independent performance of the individual enzymes, and we monitored the progress of the reaction using optical and confocal microscopy. With the systematic programming of the emulsion droplets, chemically minimal systems were realized that were capable to regulate an enzymatically-driven chemical cascade reaction over no less than three droplets of different composition.

To conclude, we systematically elucidated the osmotic pressure imbalance-driven directional mass-transfer within microdroplet networks. Aqueous emulsion droplets were programmed and tailored for a (biochemically) triggered non-reciprocal transfer of chemical substrates by compartmentalization of enzymes. We merged our findings in the realization of a multistep biochemical reaction cascade reaction across three different emulsion droplets, invoked and mediated by the parallel and independent performance of four encapsulated enzymes. Droplets capable to direct chemical reactivity and pathways give rise to speculate about artificial chemo-intelligent life-like soft materials that can perform collective functions with feedback capabilities to be used in a variety of emergent applications, such as in the development of artificial soft colloids as logical units, to gain control over multistep synthetic reaction schemes, as well as to increase the specificity of (bio-) sensors.

The authors are grateful for funding from the Max-Planck Society and through the Emmy-Noether program of the German
Research Foundation (DFG; Grant No. ZE 1121/3-1). M. P. acknowledges financial support from the Swiss National Science Foundation with the project number P2GEP2_181528. Open Access funding provided by the Max Planck Society.

\section{Conflicts of interest}

There are no conficts of interests to declare.

\section{Notes and references}

1 A. A. Hyman, C. A. Weber and F. Jülicher, Annu. Rev. Cell Dev. Biol., 2014, 30, 39.

2 S. F. Banani, H. O. Lee, A. A. Hyman and M. K. Rosen, Nat. Rev. Mol. Cell Biol., 2017, 18, 285.

3 M. Dzuricky, B. A. Rogers, A. Shahid, P. S. Cremer and A. Chilkoti, Nat. Chem., 2020, 12, 814.

4 C. Donau, et al., Nat. Commun., 2020, 11, 5167.

5 S. Deshpande, F. Brandenburg, A. Lau, M. G. F. Last, W. K. Spoelstra, L. Reese, S. Wunnava, M. Dogterom and C. Dekker, Nat. Commun., 2019, 10, 1800.

6 Y. Fujioka, et al., Nature, 2020, 578, 301.

7 M. Li, D. C. Green, J. L. Ross, B. P. Binks and S. Mann, Chem. Sci., 2011, 2, 1739.

8 R. J. R. W. Peters, M. Marguet, S. Marais, M. W. Fraaije, J. C. M. van Hest and S. Lecommandoux, Angew. Chem., Int. Ed., 2014, 53, 146.

9 X. Wang, L. Tian, H. Du, M. Li, W. Mu, B. W. Drinkwater, X. Han and S. Mann, Chem. Sci., 2019, 10, 9446.

10 M. Pavlovic, A. Plucinski, J. Zhang, M. Antonietti, L. Zeininger and B. V. K. J. Schmidt, Langmuir, 2020, 36, 1401.

11 W. M. Aumiller, B. W. Davis, N. Hashemian, C. Maranas, A. Armaou and C. D. Keating, J. Phys. Chem. B, 2014, 118, 2506.

12 Y. Chen, M. Yuan, Y. Zhang, S. Liu, X. Yang, K. Wang and J. Liu, Chem. Sci., 2020, 11, 8617.

13 J. H. van Esch, R. Klajn and S. Otto, Chem. Soc. Rev., 2017, 46, 5474.

14 S. Lach, S. M. Yoon and B. A. Grzybowski, Chem. Soc. Rev., 2016, 45, 4766.

15 Z. Sun, U. Glebe, H. Charan, A. Böker and C. Wu, Angew. Chem., Int. Ed., 2018, 57, 13810.

16 C. H. Meredith, P. G. Moerman, J. Groenewold, Y.-J. Chiu, W. K. Kegel, A. van Blaaderen and L. D. Zarzar, 2019, arXiv preprint, arXiv:1912.10102.

17 J. Liu, L. Tian, Y. Qiao, S. Zhou, A. J. Patil, K. Wang, M. Li and S. Mann, Angew. Chem., Int. Ed., 2020, 59, 6853.

18 L. Zeininger, E. Weyandt, S. Savagatrup, K. S. Harvey, Q. Zhang, Y. Zhao and T. M. Swager, Lab Chip, 2019, 19, 1327.

19 G. Villar, A. J. Heron and H. Bayley, Nat. Nanotechnol., 2011, 6, 803.

20 L. Zeininger, S. Nagelberg, K. S. Harvey, S. Savagatrup, M. B. Herbert, K. Yoshinaga, J. A. Capobianco, M. Kolle and T. M. Swager, ACS Cent. Sci., 2019, 5, 789.

21 M. Pavlovic, M. Antonietti, B. V. K. J. Schmidt and L. Zeininger, J. Colloid Interface Sci., 2020, 575, 88.

22 G. Villar, A. D. Graham and H. Bayley, Science, 2013, 340, 48.

23 S.-H. Kim, J.-G. Park, T. M. Choi, V. N. Manoharan and D. A. Weitz, Nat. Commun., 2014, 5, 3068.

24 K. Akamatsu, D. Kurita, D. Sato and S. Nakao, Langmuir, 2019, 35, 9825.

25 J.-G. Park, S.-H. Kim, S. Magkiriadou, T. M. Choi, Y.-S. Kim and V. N. Manoharan, Angew. Chem., Int. Ed., 2014, 53, 2899.

26 J. C. López-Montilla, P. E. Herrera-Morales, S. Pandey and D. O. Shah, J. Dispersion Sci. Technol., 2002, 23, 219.

27 H. González-Ochoa and J. L. Arauz-Lara, Langmuir, 2007, 23, 5289.

28 T. Tadros, P. Izquierdo, J. Esquena and C. Solans, Adv. Colloid Interface Sci., 2004, 108, 303.

29 Q. Ma, Y. Song, W. Sun, J. Cao, H. Yuan, X. Wang, Y. Sun and H. C. Shum, Adv. Sci., 2020, 7, 1903359.

30 N. Martin, ChemBioChem, 2019, 20, 2553.

31 M. Pavlovic, A. Plucinski, L. Zeininger and B. V. K. J. Schmidt, Chem. Commun., 2020, 56, 6814.

32 M. Fukuyama and A. Hibara, Anal. Chem., 2015, 87, 3562. 